\title{
Sustained Dechlorination of Vinyl Chloride to Ethene in Dehalococcoides-Enriched Cultures Grown without Addition of Exogenous Vitamins and at Low $\mathrm{pH}$
}

Luz A. Puentes Jácome, ${ }^{\dagger, \S \odot ~ P o-H s i a n g ~ W a n g, ~}{ }^{\dagger, \S, \| \odot ~ O l i v i a ~ M o l e n d a, ~}{ }^{\dagger}$ Yi Xuan (Jine-Jine) Li, ${ }^{\dagger, \perp}$ M. Ahsanul Islam, ${ }^{\dagger, \#(0)}$ and Elizabeth A. Edwards $*, \dagger, \ddagger$;

${ }^{\dagger}$ Department of Chemical Engineering and Applied Chemistry, University of Toronto, Toronto, Ontario M5S 3E5, Canada

${ }^{\ddagger}$ Department of Cell and Systems Biology, University of Toronto, Toronto, Ontario M5S 3G5, Canada

\section{Supporting Information}

ABSTRACT: Trichloroethene (TCE) bioremediation has been demonstrated at field sites using microbial cultures harboring TCE-respiring Dehalococcoides whose growth is cobalamin (vitamin $\mathrm{B}_{12}$ )-dependent. Bioaugmentation cultures grown ex situ with ample exogenous vitamins and at neutral $\mathrm{pH}$ may become vitamin-limited or inhibited by acidic $\mathrm{pH}$ once injected into field sites, resulting in incomplete TCE dechlorination and accumulation of vinyl chloride (VC). Here, we report growth of the Dehalococcoidescontaining bioaugmentation culture KB-1 in a TCE-amended mineral medium devoid of vitamins and in a VC-amended mineral medium at low $\mathrm{pH}$ (6.0 and 5.5). In these cultures, Acetobacterium, which can synthesize 5,6-dimethylbenzimidazole (DMB), the lower ligand of cobalamin, and Sporomusa are dominant acetogens. At neutral $\mathrm{pH}$, Acetobacterium supports complete TCE dechlorination by Dehalococcoides at millimolar levels with a substantial increase in cobalamin $(\sim 20$-fold $)$. Sustained dechlorination of VC to ethene was achieved at $\mathrm{pH}$ as low as 5.5. Below $\mathrm{pH} 5.0$, dechlorination was not stimulated by DMB

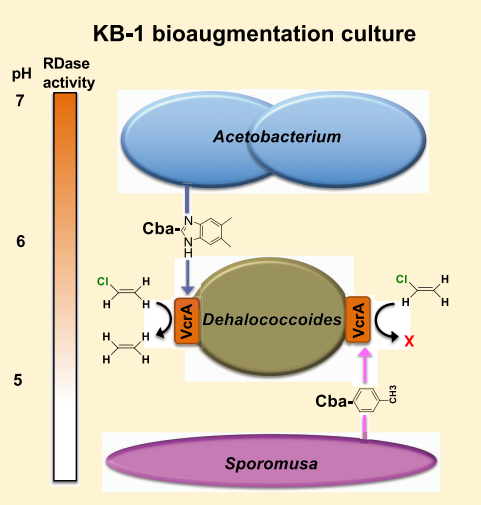
supplementation but was restored by raising $\mathrm{pH}$ to neutral. Cell-extract assays revealed that vinyl chloride reductase activity declines significantly below $\mathrm{pH} 6.0$ and is undetectable below $\mathrm{pH}$ 5.0. This study highlights the importance of cobamideproducing populations and $\mathrm{pH}$ in microbial dechlorinating communities for successful bioremediation at field sites.

\section{INTRODUCTION}

Dehalococcoides and Dehalogenimonas spp., the only known bacteria capable of reductive dechlorination of vinyl chloride (VC) to nontoxic ethene, ${ }^{1-4}$ require $\mathrm{H}_{2}$ or formate (Dehalogenimonas) as electron donor and conserve energy through organohalide respiration. Organohalide respiration depends on reductive dehalogenases (RDases) that harbor a cobamide as a prosthetic group. However, Dehalococcoides spp. cannot synthesize their own cobamide and thus import the vitamin from their surroundings. Dehalococcoides-containing cultures and isolates are typically grown in the presence of cobamides like cobalamin (vitamin $\mathrm{B}_{12}$ ). ${ }^{5,6}$ Cobamides are composed of a corrin ring, a central chelated cobalt ion, two axial ligands (upper and lower ligands), an aliphatic side chain, and a phosphodiester bond, which links the tetrapyrrole ring and the $\alpha$-ribazole (i.e., the ribonucleoside with the lower axial ligand). ${ }^{7,8}$ The best known cobamide is cobalamin, which contains 5,6-dimethylbenzimidazole (DMB) as the lower axial ligand. A recent study, ${ }^{9}$ using Dehalococcoides mccartyi strain GT, demonstrated that the vinyl chloride reductase (VcrA)mediated dechlorination of $\mathrm{VC}$ to ethene is achieved only when the lower ligand of the cobamide is DMB or 5methylbenzimidazole. ${ }^{9}$ The cobamide requirement of Dehalococcoides may be satisfied via direct cobalamin addition or via interspecies cobalamin transfer from microbial community partners. Additionally, Dehalococcoides can synthesize cobalamin via a salvage pathway that replaces the lower ligands of other cobamides with DMB. ${ }^{8-12}$ Currently, only prokaryotes are known to produce cobamides with 17 different lower axial ligand structures. ${ }^{7}$ For example, members of the family Veillonellaceae, such as Sporomusa, produce cobamides with a phenolic lower base, ${ }^{13,14}$ while members of the family Eubacteriaceae, such as Acetobacterium woodii and Eubacterium limosum, exclusively produce cobamides with a DMB lower ligand. ${ }^{15,16}$

The commercialized bioaugmentation enrichment culture KB-1 dechlorinates chlorinated ethenes, including the drycleaning solvent tetrachloroethene (PCE) and the industrial solvent trichloroethene (TCE). In the laboratory, the original KB-1 parent culture (TCE/M_1998_parent, Figure S1) is maintained with TCE (electron acceptor) and methanol (electron donor). This culture is dominated by several distinct strains of Dehalococcoides ${ }^{17,18}$ and also contains methanogens and acetogens. The historically dominant acetogens in the parent culture, which can grow using methanol as electron

Received: April 17, 2019

Revised: August 6, 2019

Accepted: August 23, 2019

Published: August 23, 2019 
donor, belong to the genera Sporomusa and Acetobacterium. ${ }^{2,19,20}$ In KB-1, Dehalococcoides consumes acetate (carbon source) and $\mathrm{H}_{2}$ (electron donor) produced by the acetogens, thus facilitating their growth. The most abundant RDase is $\mathrm{VcrA}^{21}$ whose activity is dependent on cobalamin availability. Hence, dechlorinating enrichment cultures such as KB-1 are typically grown in a medium amended with cobalamin and other vitamins to support steady dechlorination.

Another important factor influencing the performance of dechlorinating cultures is $\mathrm{pH}$. Extensive dechlorination generates $\mathrm{HCl}$, which can lead to a drop in $\mathrm{pH}$ as the buffering capacity of the medium is exhausted. A drop in $\mathrm{pH}$ to below $\mathrm{pH} 6.0$ causes a significant reduction in dechlorination rates and extent. ${ }^{22-25}$ Lower $\mathrm{pH}$ may impact the dechlorinators directly or may impact associated fermenting and cobalamin-producing organisms in the culture. Dehalococcoides populations recently studied by Yang et al. ${ }^{26}$ could not sustain reductive dechlorination at $\mathrm{pH} 5.5$, yet the reason for the loss of dechlorination activity remains unclear. In addition, the enrichment cultures grown at $\mathrm{pH} 5.5$, in which partial dechlorination of TCE to cis-dichloroethene (cis-DCE) was observed, were shown to harbor dechlorinating Sulfurospirillum, but not Dehalococcoides populations. ${ }^{26}$

Addition of vitamins and cobamides to a field site can be very expensive; vitamin $B_{12}$ (cobalamin) for field site remediation applications is sold at around 1800 USD per 100 g. $^{27}$ Similarly, $\mathrm{pH}$ adjustment at field sites can also be difficult and costly. The first objective of this study was to determine if a TCE-dechlorinating enrichment culture like KB1 , that stoichiometrically dechlorinates TCE to ethene, could be successfully maintained without exogenous vitamins. We have established a subculture of KB-1 that has sustained dechlorination of TCE to ethene without addition of vitamins for over 6 years, indicating an adequate endogenous supply of vitamins in the mixed culture. The second objective of this study was to determine if Dehalococcoides populations can sustainably dechlorinate $\mathrm{VC}$ to ethene at progressively lower $\mathrm{pH}$ and to identify the reasons for decreased activity at low $\mathrm{pH}$. Following progressive adaptation, sustained VC dechlorination to ethene was observed in KB-1 cultures maintained at $\mathrm{pH} \sim$ 6.0 and even at $\mathrm{pH} \sim 5.5$ but not observed at lower $\mathrm{pH}$. We determined that the rate of $\mathrm{VC}$ dechlorination to ethene by Dehalococcoides in KB-1 subcultures at $\mathrm{pH}$ below 5.0 was not limited by DMB or cobalamin availability but rather by a direct effect of $\mathrm{pH}$ on the VcrA-mediated dechlorination activity. The impact of these results is discussed in the context of bioremediation practice.

\section{MATERIALS AND METHODS}

Microorganisms and Chemicals. Chemical reagents were purchased through Sigma-Aldrich Canada (Oakville, ON, Canada), Fischer Scientific Canada (Ottawa, ON, Canada), and BioShop (Burlignton, ON, Canada) at the highest purity available. Gases were purchased from Praxair (Mississauga, ON, Canada). $p$-Cresolyl cobamide ${ }^{14}$ and the pure culture of Sporomusa sp. KB-1 were provided by Prof. Frank E. Löffler (University of Tennessee Knoxville).

Analytical Procedures. Sampling, DNA extraction, quantitative polymerase chain reaction (qPCR), 16S rRNA gene amplicon sequencing, cobamide extraction, and measurement protocols are detailed in the Supporting Information.

Medium Preparation. A bicarbonate-buffered FeS-reduced mineral medium ${ }^{28,29}$ was used to maintain enrichment cultures and set up new experiments. The medium was prepared either without any vitamins or with addition of one of two vitamin stock solutions: (1) a complete stock containing 11 vitamins (as described in Edwards and Grbić-Galic ${ }^{30}$ ) or (2) the same stock prepared without any vitamin $B_{12}$. The medium was autoclaved and purged while cooling with a gas mix containing $80 \% \mathrm{~N}_{2}$ and $20 \% \mathrm{CO}_{2}$. The $\mathrm{pH}$ of the medium was $6.9 \pm 0.1$. If required, the $\mathrm{pH}$ was adjusted using a $6 \mathrm{~N} \mathrm{HCl}$ solution. Except for the Sporomusa growth inhibition assay, methanol was always added as electron donor and carbon source. TCE was fed in solution with methanol, and VC (pure gas) was fed using a gastight syringe. The TCE/methanol solution was prepared at a ratio of 5:1 electron equivalents (eeq) of donor to acceptor (methanol $6 \mathrm{eeq} / \mathrm{mol}$; TCE to ethene: $6 \mathrm{eeq} / \mathrm{mol}$ ).

Vitamin-Free Enrichment Cultures. Preparation of the initial TCE-dechlorinating enrichment cultures grown and maintained without the addition of exogenous vitamins is described in the doctoral thesis of Islam (2014, available at http://hdl.handle.net/1807/90156). ${ }^{31}$ Briefly, three parallel sets of triplicate cultures $(100 \mathrm{~mL})$ were started with inoculum from a $4 \mathrm{~L}$ TCE-to-ethene-dechlorinating culture derived from the KB-1 parent culture TCE/M_1998_parent (T3MP1), i.e., an enrichment culture maintained since 1998 with TCE as acceptor and methanol (M) as donor. Cells were triple-washed in medium devoid of vitamins, i.e., centrifuged (8000 rpm, $4^{\circ} \mathrm{C}$ ), resuspended, and centrifuged again after discarding the supernatant before inoculation. The first set received medium containing the regular set of vitamins (controls), the second set received medium with all vitamins except cobalamin, and the third set received medium that was completely vitamin-free. The cultures were routinely reamended with donor and acceptor and periodically transferred into the same three medium formulations. Following the experiments described in Islam (2014), ${ }^{31}$ the cultures (i.e., triplicate $100 \mathrm{~mL}$ cultures grown at the three different medium formulations described above) were combined and scaled up to $700 \mathrm{~mL}$ and are referred to as TCE/M_Vit(+), TCE/M_Vit(-), and TCE/ M_B12(-) (Figure S1).

Low pH Enrichment Cultures. A long-term pH adaptation experiment is described in the Master's thesis of $\mathrm{Li}$ (2012, available at http://hdl.handle.net/1807/33289), ${ }^{32}$ in which transfers from TCE/M_1998_parent (T3MP1) (Figure S2) were grown over multiple feedings in media at $\mathrm{pH} 7$ (control), $\mathrm{pH}$ 6.0, and $\mathrm{pH}$ 5.5. In these cultures, the electron acceptor added was VC rather than TCE to maintain specific selection pressure for Dehalococcoides. Triplicate enrichment cultures $(50 \mathrm{~mL})$ kept at the three $\mathrm{pH}$ conditions were maintained with regular $\mathrm{VC} /$ methanol feedings for about two and a half years, prior to combining triplicates and scaling up to three $500 \mathrm{~mL}$ cultures. These scaled-up cultures are referred to as VC/M_pH7, VC/M_pH6, and VC/M_pH5.5 (Figure S2).

Growth Experiments. Two major growth experiments were performed to test the impacts of $\mathrm{pH}$ and $\mathrm{B}_{12}$ : one using $10 \%$ dilution transfers set up at $\mathrm{pH} 7.0$ and 5.5 (amended with either TCE or VC) and another using 1\% dilution transfers (amended with TCE) (see Figure S1 for experimental flow). All experiments were set up in an anaerobic chamber (Coy) with a $10 \% \mathrm{CO}_{2}, 10 \% \mathrm{H}_{2}$, and $80 \% \mathrm{~N}_{2}$ atmosphere. For the $10 \%$ dilution transfers at $\mathrm{pH} 7.0$ and 5.5 (Figure S1), inoculum from the TCE/M_B12(-) enrichment culture $(10 \mathrm{~mL}$ for TCE dechlorination assays and $5 \mathrm{~mL}$ for $\mathrm{VC}$ dechlorination 
assays) was added to autoclaved $160 \mathrm{~mL}$ narrow-necked glass serum bottles containing defined mineral medium without cobalamin $(90 \mathrm{~mL}$ for TCE dechlorination assays and $45 \mathrm{~mL}$ for VC dechlorination assays). Subsequently, the serum bottles were sealed with rubber stoppers and crimped. The electron donor (methanol) and electron acceptor (TCE or VC) were added in the same way as for the enrichment cultures, at a ratio of 5 to 1 electron equivalents of donor to acceptor, unless otherwise specified. If necessary, additional electron donor was added to ensure complete dechlorination. Once the TCE transfers set up at $\mathrm{pH} 5.5$ had partially dechlorinated the added TCE to ethene, they were subsequently aliquoted to six $10 \mathrm{~mL}$ glass vials to a final volume of $5 \mathrm{~mL}$ each, sealed with rubber stoppers and crimped. DMB $(10 \mu \mathrm{M})$ was added to two bottles, and the $\mathrm{pH}$ was adjusted to 7.0 in another two bottles using a saturated bicarbonate solution $(\sim 1.3 \mathrm{M})$. The remaining two bottles were kept as controls at $\mathrm{pH}$ 5.5. The growth of Sporomusa and Acetobacterium in the absence of TCE was investigated in $10 \%$ dilution transfers from the TCE/ M_B12(-) culture that were fed methanol only (Figure S1).

The $1 \%$ dilution transfer experiments (Figure S1) were initiated to minimize cobalamin and DMB carry-over. In these experiments, the conditions were similar to those of the $10 \%$ dilution transfers, except that the inoculum $(1 \mathrm{~mL})$ from the TCE/M_Vit(-) was first pelleted down, washed twice with sterilized defined (cobalamin-free) medium, resuspended in 1 $\mathrm{mL}$ of the same cobalamin-free medium, and then added to serum bottles containing $99 \mathrm{~mL}$ of cobalamin-free medium.

Cell-Extract Dehalogenase Activity Assay. The cellextract dehalogenase activity assay was performed following previously established protocols under anaerobic conditions. ${ }^{33}$ All reagents were pre-reduced or kept anaerobically before the assays. Briefly, $100 \mathrm{~mL}$ of the TCE/M_B12(-) culture was harvested and pelleted down by centrifugation at $9000 \mathrm{~g}$ for 20 min at $4{ }^{\circ} \mathrm{C}$ under anaerobic conditions. The supernatant was removed and the pellets were resuspended in $0.3 \mathrm{~mL}$ of 100 $\mathrm{mM}$ Tris- $\mathrm{HCl}$ buffer ( $\mathrm{pH} 7.5$ ) containing $12.5 \mathrm{mM} \mathrm{NaCl}$, $2.5 \%$ glycerol, and $1 \%$ digitonin (detergent). The suspensions were loaded into a $2 \mathrm{~mL}$ O-ring-capped plastic microcentrifuge tube containing $50 \mathrm{mg}$ of glass beads (particle size $\leq 106 \mu \mathrm{m}$ ). To lyse the cells, the tube was vortexed at maximum intensity for $2 \mathrm{~min}$ and incubated on ice for $1 \mathrm{~min}$. The step was repeated four times. The cell lysate was then centrifuged at $16000 \mathrm{~g}$ for $15 \mathrm{~min}$ at $4{ }^{\circ} \mathrm{C}$ to remove cell debris, beads, and unbroken cells, and the supernatant was used in the assays. The dehalogenase activity assays $(1 \mathrm{~mL})$ were performed in capped $2 \mathrm{~mL}$ glass vials. Tris- $\mathrm{HCl}$ buffer $(50 \mathrm{mM})$ was used for the assays at $\mathrm{pH} 6.0$ and 7.0, and sodium acetate-acetic acid

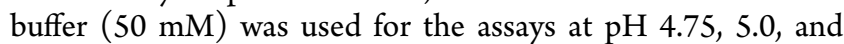
5.5. $\mathrm{Ti}(\mathrm{III})$ citrate-reduced methyl viologen $(1 \mathrm{mM})$ was used as the artificial electron donor, and cis-DCE $(0.5 \mathrm{mM})$ was used as the electron acceptor; these were added to the buffers before the addition of cell extracts. The cell extracts were added into the vials to a final protein concentration of $10 \mu \mathrm{g} /$ $\mathrm{mL}$ to start the reaction. The vials were immediately capped, incubated for $2 \mathrm{~h}$, and sampled for analysis of dechlorination products via gas chromatography (see Supporting Information). Protein concentration was determined using a microplate Bradford (Sigma-Aldrich Bradford Reagent) protein assay with bovine serum albumin standards.

Sporomusa Growth Inhibition Assays. For this experiment, $\mathrm{Na}_{2} \mathrm{~S}(0.2 \mathrm{mM})$, instead of $\mathrm{FeS}$, was used as the reductant in the medium to avoid turbidity and facilitate cell density measurements; resazurin was excluded from the medium to avoid interference from the pink color that develops during cell density measurements after exposure to air. In an anaerobic chamber, the active Sporomusa sp., strain $\mathrm{KB}-1$ pure culture $\left(2 \mathrm{~mL} ; \mathrm{OD}_{600 \mathrm{~nm}} \sim 1\right)$, was transferred into 2 $\mathrm{mL}$ sterile O-ring-capped plastic microcentrifuge tubes and pelleted down by centrifugation at $10000 \mathrm{~g}$ for $10 \mathrm{~min}$ at room temperature. The supernatants were removed, and the pellets were washed with a sterile cobalamin-free defined medium (1 $\mathrm{mL})$ twice. Afterward, the pellets were resuspended in sterile defined media containing varying cobalamin concentrations $(0$, $5,10,25$, and $50 \mu \mathrm{g} / \mathrm{L})$ to a final cell density $\left(\mathrm{OD}_{600 \mathrm{~nm}}\right)$ of 0.04 (as determined by the no-substrate control included in the experiment); $5 \mathrm{~mL}$ of the resuspended cells at each cobalamin concentration was transferred into the sterile $10 \mathrm{~mL}$ rubber-stopper-sealed glass tubes by sterile plastic syringes and needles. The tubes were then purged with $20 \% \mathrm{CO}_{2} / 80 \% \mathrm{~N}_{2}$ to remove $\mathrm{H}_{2}$, followed by the addition of methanol to a final concentration of $20 \mathrm{mM}$. In some assays, $\mathrm{H}_{2}(20 \%$ in head space), formate $(10 \mathrm{mM})$, or lactate $(10 \mathrm{mM})$ was added. The glass tubes were incubated at $30{ }^{\circ} \mathrm{C}$ for 1 day, and $0.2 \mathrm{~mL}$ of each culture was sampled and loaded into a 96-well optical plate (Falcon). The cell density in each well was determined using a TECAN Infinite M200 plate reader at $600 \mathrm{~nm}$.

Accession Numbers. 16S rRNA amplicon sequences have been deposited into NCBI under bioproject PRJNA376155 SRA study SRP144609 accession numbers SRR9839478-86. The draft genomes of Sporomusa sp. KB-1 and Acetobacterium sp. KB-1 are available in IMG under genome ID 2512047088 and NCBI under accession number CP030040, respectively.

\section{RESULTS AND DISCUSSION}

We first describe the results of the long-term cultivation experiment with cultures grown with TCE in mineral minimal medium without exogenous vitamins and without cobalamin. Then, we describe the results of the long-term cultivation experiment with cultures grown with $\mathrm{VC}$ at $\mathrm{pH} 7.0,6.0$, and 5.5. Next, we describe the microbial community of these cultures as determined by $16 \mathrm{~S}$ rRNA gene amplicon sequencing and describe the hypotheses that led to subsequent experiments to investigate the dynamic relationship between the acetogens in this culture in light of Dehalococcoides cobamide dependence and the sensitivity of reductive dechlorination to $\mathrm{pH}$ below 6.0 .

Reductive Dechlorination of TCE to Ethene in Enrichment Cultures Grown without Exogenous Vitamins. Dechlorination of TCE to ethene was sustained over 800 days (Figure 1) in all of the cultures, TCE/M_Vit $(+)$, TCE/M_B12(-), and TCE/M Vit $(-)$, regardless of the type of vitamin amendment. In general, the cultures with or without exogenous vitamins performed similarly (Figure 1A,C). The average long-term dechlorination rates for these three cultures, taken as the slope of the linear regression of the data over 800 days as shown in Figure 1, were $69 \pm 2.4,66 \pm 2.2$, and $57 \pm$ $1.3 \mu \mathrm{mol} \mathrm{Cl}{ }^{-}$released/L/d, respectively. The average dechlorination rate for TCE/M Vit(-) is slightly lower than that for TCE/M_Vit(+). Although this could be related to the high metabolic cost associated with the synthesis of the required cobamides, in practice, these differences are within the expected variability in dechlorination rates that may be observed during this type of experiments. Altogether, these long-term data demonstrate that the entire vitamin require- 
Dechlorination of TCE to ethene A. TCE/M_Vit(+)
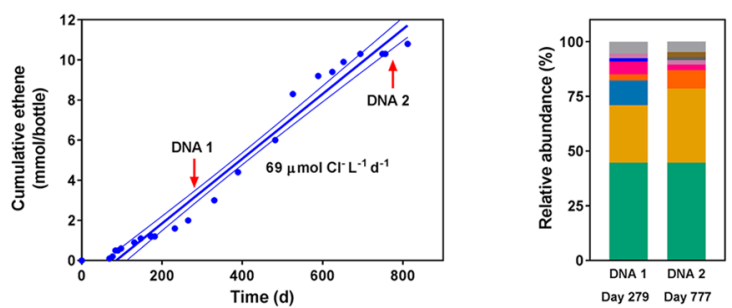

B. TCE/M_B12(-)
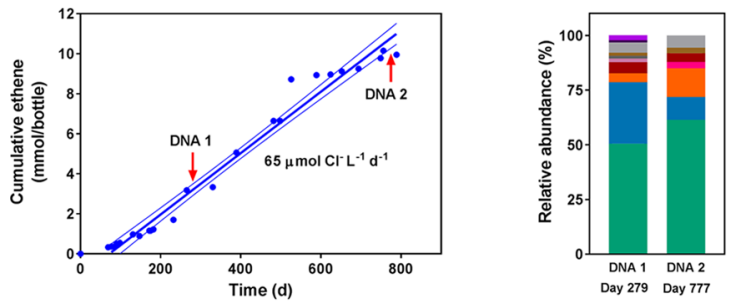

C. TCE/M_Vit(-)
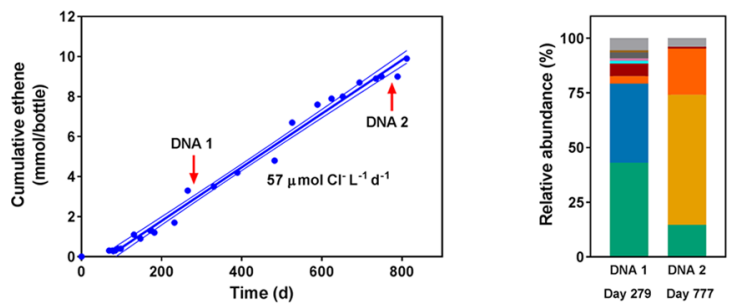

Microbial groups:

\begin{tabular}{|c|c|c|c|}
\hline - & genus_Desulfosporosinus & - & order_Bacteriodales \\
\hline & genus_C1_B005 & - & genus_Treponema \\
\hline$=$ & $<1 \%$ & - & genus_Methanoregula \\
\hline - & genus_E7 & - & genus_Dehalobacter \\
\hline & genus_Thermacetogenium & - & genus_Acetobacterium \\
\hline & class_SJA-28 & $=$ & genus_Sporomusa \\
\hline & $\begin{array}{l}\text { class_OP5 } \\
\text { family_Porphyromonadaceae }\end{array}$ & $=$ & genus_Dehalococcoides \\
\hline
\end{tabular}

Figure 1. Long-term and sustained dechlorination of TCE to ethene (left) and corresponding microbial community composition shifts (right) for the scaled-up TCE- and methanol-fed enrichment cultures TCE/M_Vit(+) (A), TCE/M_Vit(-) (B), and TCE/M_B12(-) (C). TCE was fed every 4-6 weeks, at a nominal concentration of 1.4-1.5 mM. Dechlorination rates obtained from linear regressions are shown with $95 \%$ confidence intervals; the $R^{2}$ values of the regressions are $0.98,0.99$, and 0.98 for (A), (B), and (C), respectively. On the right, two amplicon sequencing events are shown for DNA extracted on day 279 and day 777 .

ments of the culture could be met by organisms present in the KB-1 culture.

Reductive Dechlorination of VC to Ethene in Enrichment Cultures Grown at $\mathrm{pH} 6.0$ and $\mathrm{pH}$ 5.5. The reductive dechlorination of $\mathrm{VC}$ to ethene in the cultures $\mathrm{VC} /$ $\mathrm{M} \mathrm{pH7,} \mathrm{VC/M} \mathrm{pH6,} \mathrm{and} \mathrm{VC/M} \mathrm{pH5.5} \mathrm{is} \mathrm{shown} \mathrm{in} \mathrm{Figure}$ $2 \mathrm{~A}$. The observed dechlorination rates for these cultures, taken as the slope of the linear regression of the data set shown for each culture $(\sim 400$ days $)$, are $7.9 \pm 0.29,8.8 \pm 0.25$, and $2.0 \pm$ $0.08 \mu \mathrm{mol} \mathrm{Cl}{ }^{-}$released/L/d, respectively. The enrichment culture originally set at $\mathrm{pH}$ 6.0, described in the Master's thesis of $\mathrm{Li}(2012),{ }^{32}$ initially dechlorinated VC more slowly than the culture at $\mathrm{pH} 7.0$ but eventually maintained a rate comparable to that of the enrichment culture maintained at $\mathrm{pH} 7.0$ (Figure 2). The average dechlorination rate for $\mathrm{VC} / \mathrm{M} \_\mathrm{pH} 6$ is slightly higher than that for $\mathrm{VC} / \mathrm{M} \_\mathrm{pH}$, yet in practice these differences may be due to the nature of the experimental set up, i.e., occasional small ethene leaks during sampling in the $\mathrm{pH} 7.0$ bottle or differences in feeding amounts (gaseous VC is fed directly), or may be related to changes in the culture composition through the period of adaptation to $\mathrm{pH}$ 6.0. In the culture maintained at $\mathrm{pH} 5.5$ (see Figure $\mathrm{S} 3$ for $\mathrm{pH}$ measurements), the observed dechlorination and ethene production rates were about 4 times lower than those at $\mathrm{pH}$ 7.0 and 6.0. The maintenance of low $\mathrm{pH}$ batch cultures required careful monitoring as acid buildup from dechlorination progressively lowered the $\mathrm{pH}$; at low $\mathrm{pH}$, the carbonate buffering system is not as robust as at neutral $\mathrm{pH}$. Overall, we found that the enrichment culture $\mathrm{KB}-1$ was able to dechlorinate $\mathrm{VC}$ to ethene at $5.5 \leq \mathrm{pH} \leq 7.0$ (Figure $2 \mathrm{~A}$ ) and that this activity was mediated by Dehalococcoides (Figure 2B), which is different from the observations reported by Yang et al. ${ }^{26}$ Perhaps, certain Dehalococcoides populations are more resistant to low $\mathrm{pH}$ than others.

Microbial Community Composition Revealed by Amplicon Sequencing. Two snapshots of the microbial community composition in the enrichment cultures used in this study are shown in the right-hand panels in Figures 1 and 2. As expected, Dehalococcoides was the dominant genus in these cultures, accounting for about $25-50 \%$ of the total microbial population based on $16 \mathrm{~S}$ rRNA gene copies. As previously reported, ${ }^{2,19,20}$ the two major acetogens were Acetobacterium and Sporomusa. On day 279 (first DNA sampling), Sporomusa was more abundant in the enrichments amended with vitamins (TCE/M_Vit $(+)$ ) than in the nonamended ones. Sporomusa was also more abundant than Acetobacterium in the sample from VC/M pH5.5. The relative abundance of Sporomusa increased in the TCE/M Vit(-) enrichment cultures between day 279 and day 777 . We also noted that the $\mathrm{pH}$ in this culture, TCE/M Vit $(-)$, and in the TCE/M_Vit(+) had fallen to $4.7-4.8^{-}$(as a result of progressive $\mathrm{HCl}$ accumulation from dechlorination). The $\mathrm{pH}$ was adjusted back to 7.0, but perhaps this $\mathrm{pH}$ drop, which likely occurred over a period of 2-4 weeks after feeding, had already affected the microbial community. Nevertheless, these observed differences in the relative abundance of Acetobacterium and Sporomusa led us to propose two hypotheses: (1) Sporomusa outcompetes Acetobacterium at $\mathrm{pH}$ below $\mathrm{pH} 6.0$ and (2) Sporomusa outcompetes Acetobacterium when exogenous vitamins (particularly vitamin $B_{12}$ ) are present. To test the $\mathrm{pH}$ hypothesis further, we carried out a point biserial (pb) correlation analysis between the microbial abundance data and $\mathrm{pH}$ above or below 6.0. We used the data shown in Figures 1 and 2, along with two samples from the parent KB-1 culture and a VC/methanol-fed KB-1 subculture (see Table S2). The relative abundance of Acetobacterium was negatively correlated $\left(r_{\mathrm{pb}}=-0.75\right)$ with $\mathrm{pH}<6.0$ (anytime within 3 weeks prior to DNA extraction). In contrast, the relative abundance of Sporomusa was positively correlated $\left(r_{\mathrm{pb}}=0.77\right)$ with $\mathrm{pH}>6.0$. Acetobacterium also seemed to be more abundant in cultures without added vitamin $B_{12}$ (Figure $1 B, C$ ). Additional growth experiments (described below) were initiated to further investigate these trends.

Dechlorination of TCE at $\mathrm{pH} 7.0$ and $\mathrm{pH} 5.5$ in Cultures Grown without Exogenous Cobalamin (Vitamin $B_{12}$ ). Using a $10 \%$ inoculum from culture TCE/ M B12(-), we examined the growth of Dehalococcoides, Acetobacterium, and Sporomusa (measured by qPCR) during the dechlorination of TCE to ethene in parallel experiments at 

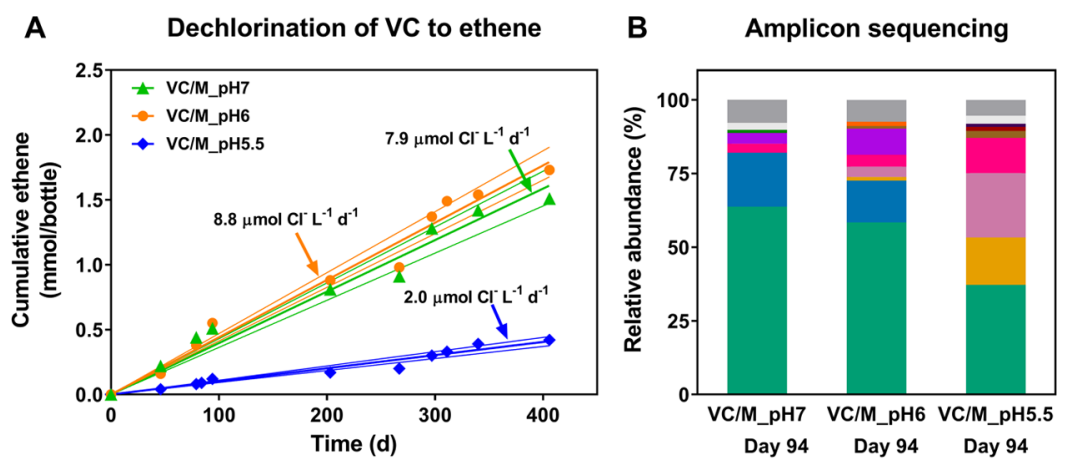

Microbial groups

\begin{tabular}{|c|c|c|c|}
\hline & $<1 \%$ & genus_Methanoregula & class_SJA-28 \\
\hline & genus_E7 & order_Bacteriodales & genus_Sporomusa \\
\hline ש & genus_Desulfovibrio & genus_Desulfosporosinus & genus_Acetobacterium \\
\hline ש & genus_Longilinea & genus_Treponema & genus_Dehalococcoides \\
\hline - & genus_Blvii29 & & \\
\hline
\end{tabular}

Figure 2. Long-term and sustained dechlorination of $\mathrm{VC}$ to ethene in enrichment cultures maintained at $\mathrm{pH} 7.0,6.0$, and $5.5 \pm 0.2$ (A) and their corresponding microbial community composition on day 94 (B). Dechlorination rates obtained from linear regressions with $95 \%$ confidence intervals are shown; the $R^{2}$ values of the linear regressions are $0.96,0.97$, and 0.96 , for the $\mathrm{pH} 7.0, \mathrm{pH} 6.0$, and $\mathrm{pH} 5.5$ enrichment cultures, respectively.

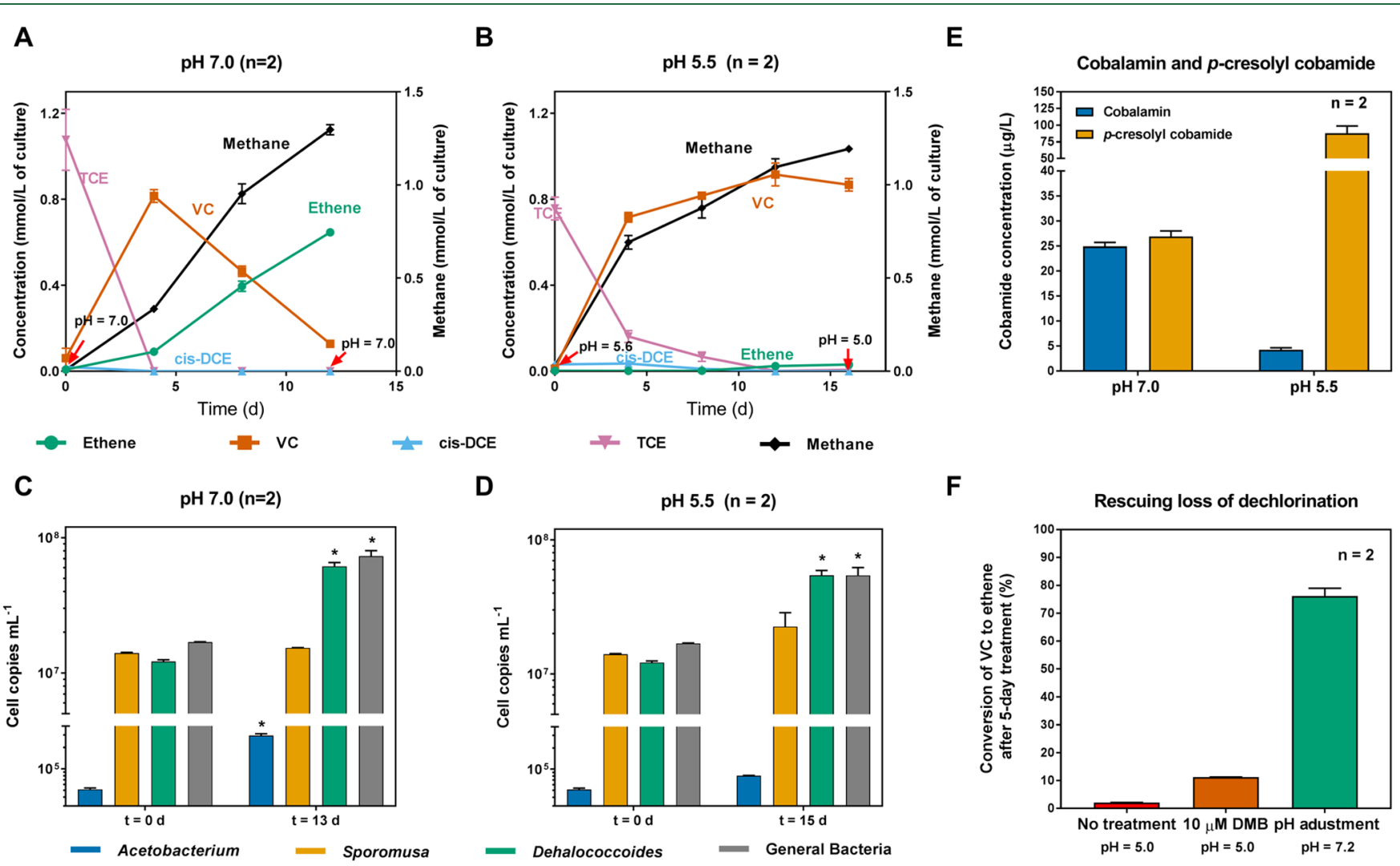

Figure 3. TCE dechlorination profiles in 10\% dilution transfers of the TCE/M_B12(-) at pH 7.0 (A) and pH 5.5 (B) and associated cell and corrinoid abundances. Corresponding cell abundances per $\mathrm{mL}$ of culture as determined by qPCR are shown for $\mathrm{pH} 7.0$ in panel (C) and pH $5.5 \mathrm{in}$ panel (D). Cobalamin and $p$-cresolyl cobamide concentrations are shown in panel (E). Attempts to reactivate $\mathrm{pH} 5.0$ culture samples from day 16 in panel B with DMB addition or $\mathrm{pH}$ adjustment are shown in panel (F). In panels (A) and (B), initial and final pH measurements are shown. Error bars correspond to the range of duplicate experimental bottles except for the qPCR samples at $t=0$ day in which a single sample was measured and error bars correspond to the range for technical duplicates. The * symbol indicates growth greater than 5-fold as compared to the measurements obtained at $t=0$ day. In (E), cobalamin and $p$-cresolyl cobamide were measured at $t=16$ days (final time point for the transfers set up at $\mathrm{pH} 5.5$ ). In $(\mathrm{F})$, the $\mathrm{pH}$ indicated below the bars corresponds to the $\mathrm{pH}$ measured in the bottles after DMB addition or $\mathrm{pH}$ adjustment; in the "no treatment" and "10 $\mu \mathrm{M}$ DMB" bottles, the $\mathrm{pH}$ fell from 5.5 to 5.0 as a result of dechlorination.

$\mathrm{pH} 7.0$ and 5.5. Gene copies per $\mathrm{mL}$ were converted to cells per $\mathrm{mL}$ using the number of $16 \mathrm{~S}$ rRNA gene copies found in the genomes of Acetobacterium sp. KB-1 (5 copies, draft genome in NCBI under accession number CP030040) and Sporomusa sp. KB-1 (13 copies, draft genome in IMG under genome ID: 2512047088). Dehalococcoides only possesses a 
Table 1. Cell growth, Yield, and Cobalamin Production Measured in a 1\% Dilution Transfer of the TCE/M_Vit(-) Enrichment Culture Dechlorinating TCE to Ethene in the Absence of Any Added Vitamins ${ }^{a}$

\begin{tabular}{lccc}
\multicolumn{1}{c}{ measurement } & inoculum $(t=0$ day $)$ & final $(t=60$ days $)$ & yield $\left(\right.$ cells per $\mu$ mol $\mathrm{Cl}^{-}$released $)$ \\
Dehalococcoides $($cells $/ \mathrm{mL})$ & $2.4 \pm 0.4 \times 10^{7}$ & $1.7 \pm 0.05 \times 10^{8}$ & $1.1 \pm 0.04 \times 10^{8}$ \\
Acetobacterium $($ cells $/ \mathrm{mL})$ & $5.7 \pm 0.2 \times 10^{3}$ & $3.6 \pm 0.04 \times 10^{5}$ & 7.3 \\
total cobalamin $(\mathrm{ng} / \mathrm{L})$ & $40 \pm 5$ & $750 \pm 140$ & 63
\end{tabular}

${ }^{a}$ Dechlorination data is shown in Figure S5. Cell growth and cobalamin concentrations are reported as the mean \pm range of duplicate measurements.

single copy of the $16 \mathrm{~S}$ rRNA gene per genome. As shown in Figure $3 \mathrm{~A}, \mathrm{~B}$, complete dechlorination of TCE to ethene was only observed at $\mathrm{pH}$ 7.0. At $\mathrm{pH} 5.5$, TCE was dechlorinated to $\mathrm{VC}$, but $\mathrm{VC}$ was not further dechlorinated to ethene. In these bottles, the dechlorination of TCE $(0.75 \mathrm{mM}$ per bottle) to $\mathrm{VC}$ at $\mathrm{pH} 5.5$ caused the $\mathrm{pH}$ to drop to 5.0. This drop in $\mathrm{pH}$ likely impacted the ability of the Dehalococcoides population to dechlorinate VC to ethene. Nonetheless, an increase in the cell numbers of Dehalococcoides per $\mathrm{mL}$ of culture (as compared to the measurements obtained at $t=0$ day) was observed at both pH 7 and 5.5 (Figure 3C,D; Table S3); at pH 5.5, the growth of Dehalococcoides was proportional to observed dechlorination (two-thirds of the electron acceptor equivalents were consumed at $\mathrm{pH} 5.5$ compared to $\mathrm{pH} 7$ ). In the transfers set up at $\mathrm{pH}$ 7.0, there was a 5-fold increase of Acetobacterium, but no increase of Sporomusa. In the transfers set up at $\mathrm{pH} 5.5$, Acetobacterium and Sporomusa both showed a small 2 -fold increase (Figure 3C,D; Table S3). Slow to no growth of Acetobacterium at $\mathrm{pH} 5.5$ was expected as the $\mathrm{pH}$ growth range for other Acetobacterium spp. is between 6.0 and 8.0, with a $\mathrm{pH}$ optimum around $7.0 .^{34-37}$ In contrast, the $\mathrm{pH}$ growth optimum for Sporomusa spp. is between 6.2 and $7.4,{ }^{38}$ with a $\mathrm{pH}$ growth range as low as 5.5 and as high as $8.7 .^{38-40}$ For Sporomusa silvacetica, the closest relative of Sporomusa sp. KB1 , relatively rapid growth was reported for $\mathrm{pH}$ between 5.5 and 7.5. ${ }^{39}$

Cobamide Profile in Cultures Grown at pH 7.0 and 5.5. Since Acetobacterium spp. are reported to be cobalamin producers, ${ }^{15,16}$ we hypothesized that the amount of cobalamin in the cultures would be proportional to the relative abundance of the Acetobacterium population. Thus, we extracted the cobamides from the aforementioned KB-1 transfers grown at $\mathrm{pH} 7.0$ and at $\mathrm{pH}$ 5.5, respectively (Figure 3E; mass spectroscopy (MS) spectra in Figure S4). Consistently, in the cultures grown at $\mathrm{pH} 5.5$ in which $\mathrm{VC}$ accumulated, the measured concentration of $p$-cresolyl cobamide was $\sim 88 \mu \mathrm{g} / \mathrm{L}$, which is $\sim 20$ times higher than the measured concentration of cobalamin $(\sim 4 \mu \mathrm{g} / \mathrm{L})$. In contrast, in the cultures grown at $\mathrm{pH}$ 7.0, in which VC was completely dechlorinated and the growth of Acetobacterium was measureable (5-fold increase), the average concentrations of p-cresolyl cobamide and cobalamin are comparable $(\sim 25 \mu \mathrm{g} / \mathrm{L})$. The higher cell density of Acetobacterium and higher cobalamin concentration in the cultures grown at $\mathrm{pH} 7.0$, as compared to the cultures grown at $\mathrm{pH}$ 5.5, support our hypothesis that Acetobacterium is a DMB/ cobalamin producer in the KB-1 enrichment cultures. To further confirm that Acetobacterium sp. strain KB-1 can supply $\mathrm{DMB} /$ cobalamin to Dehalococcoides, we inoculated medium without cobalamin with a $1 \%$ transfer from the culture TCE/ M_Vit(-). In the previous experiment (10\% dilution transfers), some DMB or cobalamin could have carried over with the inoculum. For this experiment, the inoculum was centrifuged and washed twice with anaerobic mineral medium devoid of vitamins to remove cobalamin and DMB carry-over. After the duplicate KB-1 cultures had dechlorinated $\sim 0.5 \mathrm{mM}$ TCE to ethene $\left(1.34 \mathrm{mM} \mathrm{Cl}^{-}\right.$released), we harvested one of the cultures (dechlorination data shown in Figure S5) and measured cobalamin concentrations. The measured concentration $(\sim 750 \mathrm{ng} / \mathrm{L}, 0.55 \mathrm{nM})$ is almost 20 -fold higher than the observed concentration before TCE dechlorination occurred (Table 1). Nevertheless, it is surprising that nanomolar levels of cobalamin could support the dechlorination of TCE to ethene. For this experiment, the yield of Dehalococcoides, $1.1 \times 10^{8}$ cells per $\mu \mathrm{mol} \mathrm{Cl}{ }^{-}$released, is in the same order of magnitude as previously published yields of Dehalococcoides pure cultures, which range between $1.5 \times 10^{7}$ and $2.9 \times 10^{8}$ cells per $\mu \mathrm{mol} \mathrm{Cl}{ }^{-}$released. ${ }^{41}$ Moreover, the cell density for Acetobacterium increased by 63-fold (Table 1).

In KB-1, dominant methanogens from the Methanomicrobiales, such as Methanoregula, may produce factor $\mathrm{III}^{42}$ (with 5hydroxybenzimidazole as lower ligand) while Desulfosporosinus and Dehalobacter may produce purinyl-cobamide as predicted by Yan et al. ${ }^{7}$ Sulfate reducers like Desulfovibrio, although not in high abundance, may produce cobamides with guanine or hypoxanthine as lower ligand, as demonstrated previously for Desulfovibrio vulgaris. ${ }^{43}$ These cobamides, in any case, are not functionally equivalent to cobalamin for $\mathrm{VcrA}$, which catalyzes the dechlorination of $\mathrm{VC}$ to ethene. In fact, supplementing other cobamides like factor III, phenyl cobamide or purinylcobamides, to Dehalococcoides isolate cultures that synthesize the VcrA and BvcA enzymes (which can dechlorinate VC to ethene) resulted in incomplete dechlorination and $\mathrm{VC}$ accumulation. ${ }^{9}$ Nevertheless, Dehalococcoides is able to synthesize cobalamin via a salvage pathway that replaces the lower ligands of other cobamides with DMB., ${ }^{9,13}$

Relationship between Acetobacterium and Dehalococcoides during TCE Reductive Dechlorination. As demonstrated earlier, the growth of Acetobacterium in KB-1 is correlated to an increase in cobalamin content, which supports efficient dechlorination of VC to ethene (Table 1). Analyses of the KB-1 metagenome (NCBI accession PRJNA376155) are consistent with this observation, revealing that only Acetobacterium sp. strain KB-1 contains a complete anaerobic DMB biosynthesis operon (bzaABCDE; Figure S6). The operon arrangement and gene annotation are identical to those of $E$. limosum strain ATCC 10825 and A. woodii, two well-known cobalamin producers ${ }^{16,44,45}$ (Figure S6); the encoded proteins are most similar to the ones found in Acetobacterium dehalogenans (Table S4).

We were next interested in studying the growth of Acetobacterium in the absence of dechlorination to further understand the relationship between Acetobacterium and Dehalococcoides. We performed a $10 \%$ dilution transfer of the culture TCE/M B12(-) into medium free of vitamins amended with methanol but not TCE, at both $\mathrm{pH} 7.0$ and 5.5. Contrary to our expectation, we did not observe growth of 
Acetobacterium (Figure S7). Measurable growth (an order of magnitude) was observed only for Sporomusa, regardless of $\mathrm{pH}$. Dehalococcoides was not expected to grow at either $\mathrm{pH}$ tested, yet we expected to observe measurable growth for Acetobacterium analogous to the growth that we observed at pH 7.0 with added TCE (Figure 3C). While it is clear that $\mathrm{DMB} /$ cobalamin produced by Acetobacterium is beneficial to Dehalococcoides, any potential mechanism that may facilitate the growth of Acetobacterium during active dechlorination by Dehalococcoides remains to be further explored.

When cobalamin is absent at field sites, VC dechlorination by Dehalococcoides can be expected to be highly dependent on the biosynthesis of $\mathrm{DMB} /$ cobalamin by cobalamin-producing organisms such as Acetobacterium. The role that native Acetobacterium and other cobamide-producing populations have at chlorinated ethene-contaminated sites should be investigated. Environmental practitioners working on biostimulation via cobalamin addition may find it beneficial to first assess if native cobalamin-producing populations may support in situ VC dechlorination. Acetobacterium and other cobamideproducing organisms can be quantified, via qPCR of the $16 \mathrm{~S}$ rRNA gene for example, in addition to Dehalococcoides $16 \mathrm{~S}$ rRNA genes, Dehalococcoides RDase genes, and 16S rRNA genes for other dechlorinating organisms. Cobamide-producing organisms such as Acetobacterium may indeed serve as additional biomarkers for successful field site reductive dechlorination of VC.

Cobalamin in Methanol-Fed Enrichment Cultures May Inhibit Sporomusa. According to our findings, when enrichment cultures are starved of TCE (Figure S7) or experience low $\mathrm{pH}$ (Figures $2 \mathrm{~B}$ and $3 \mathrm{D}$ ), Sporomusa growth is favored. Sporomusa can also efficiently grow at neutral $\mathrm{pH}$ as shown in Figure S7. An earlier study ${ }^{46}$ reported that the addition of $10 \mu \mathrm{M} \mathrm{DMB}(\sim 1400 \mu \mathrm{g} / \mathrm{L})$ to growth medium inhibited the growth of Sporomusa on methanol since the methanol methyltransferase requires either phenyl cobamide or $p$-cresolyl cobamide as a cofactor. ${ }^{46-48}$ We hypothesized that cobalamin, the cobamide with DMB as the lower ligand, may also inhibit the growth of Sporomusa on methanol. When 50 $\mu \mathrm{g} / \mathrm{L}$ cobalamin was added to the medium, the growth of Sporomusa was completely inhibited (Figure 4). When $25 \mu \mathrm{g} / \mathrm{L}$ cobalamin was added to the medium, the growth of Sporomusa

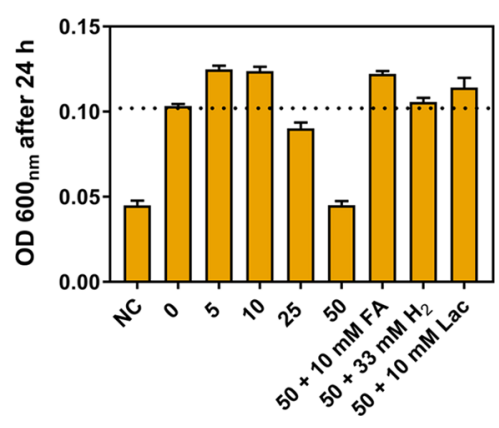

Figure 4. Inhibition of Sporomusa growth on methanol by cobalamin. Assays tested $0,5,10,25$, and $50 \mu \mathrm{g} / \mathrm{L}$ cobalamin $+20 \mathrm{mM}$ methanol; $50 \mu \mathrm{g} / \mathrm{L}$ cobalamin $+10 \mathrm{mM}$ sodium formate; $50 \mu \mathrm{g} / \mathrm{L}$ cobalamin $+33 \mathrm{mM}$ (nominal) hydrogen gas; and $50 \mu \mathrm{g} / \mathrm{L}$ cobalamin $+10 \mathrm{mM}$ sodium lactate $(\mathrm{Lac})$. Growth was measured using $\mathrm{OD}_{600 \mathrm{~nm}}$ after $24 \mathrm{~h}$. NC stands for the no-substrate control. Above the dotted line, no inhibition is observed. Error bars represent the standard deviation of triplicate experiments. was lower relative to the control. This is about the same concentration as measured in the $\mathrm{pH} 7.0$ experiment shown in Figure 3 in which the growth of Sporomusa was not apparent (Figure 3C). The growth of Sporomusa was not inhibited by cobalamin when grown with $\mathrm{H}_{2}$, formate, or lactate instead of methanol (Figure 4), suggesting that the inhibition by cobalamin is specific to growth on methanol, which requires the $p$-cresolyl cobamide- or phenyl cobamide-specific methanol methyltransferase. It is possible that the use of methanol as the sole electron donor in the KB-1 enrichment cultures may play a role in the enrichment of the $\mathrm{DMB} /$ cobalamin-producing Acetobacterium population, whose production of cobalamin represses the growth of the $p$-cresolyl cobamide-producing Sporomusa population.

Stalled Dechlorination of VC to Ethene Was Restored after pH Adjustment. Our data revealed that the population of cobalamin/DMB-producing Acetobacterium is much less abundant in the dilution transfer cultures grown at $\mathrm{pH} 5.5$ than in those grown at $\mathrm{pH} 7.0$ (Figure 3), suggesting that Dehalococcoides may have faced cobalamin shortage to produce the functional form of VcrA for VC dechlorination. Given that Dehalococcoides can salvage cobalamin using $p$-cresolyl cobamide and $\mathrm{DMB},{ }^{12}$ we wanted to test if $\mathrm{DMB}$ supplementation $(10 \mu \mathrm{M} ; \sim 1400 \mu \mathrm{g} / \mathrm{L})$ alone could rescue the loss of VC dechlorination in these transfers. As shown in Figure 3F, DMB supplementation did not restore the dechlorination of $\mathrm{VC}$ to ethene at $\mathrm{pH}$ 5.5. However, increasing $\mathrm{pH}$ to 7.0 led to $80 \%$ conversion of $\mathrm{VC}$ to ethene in 5 days. This suggests that incomplete VC dechlorination by Dehalococcoides was not due to DMB shortage but due to acidic $\mathrm{pH}$.

Effect of $\mathrm{pH}$ on the Dehalococcoides-Mediated Reductive Dechlorination of VC in 10\% Dilution Transfers Amended with VC. Analogous to the 10\% dilution transfer experiments set up at $\mathrm{pH} 7.0$ and 5.5 with TCE as electron acceptor, a $10 \%$ dilution transfer experiment with the culture TCE/M_B12(-), maintained at circumneutral $\mathrm{pH}$, was performed to specifically test the dechlorination of $\mathrm{VC}$ at different $\mathrm{pH}$ values: 7.0, 6.3, 5.5, and 5.5 with 10 $\mu \mathrm{M}$ DMB. As shown in Figure 5A, complete VC dechlorination to ethene only occurred at $\mathrm{pH} 7.0$ and $\mathrm{pH}$ 6.0. The dechlorination of $\mathrm{VC}$ to ethene was slower in the transfers set up at $\mathrm{pH} 5.5$, but ethene was produced until the $\mathrm{pH}$ decreased below 5.5. Dehalococcoides growth was observed at each $\mathrm{pH}$ (Table 2). Even though the enrichment culture TCE/ M_B12(-) is grown at circumneutral $\mathrm{pH}$, Dehalococcoides was still able to grow and dechlorinate $\mathrm{VC}$ to ethene at a $\mathrm{pH}$ as low as 5.5. The addition of $10 \mu \mathrm{M} \mathrm{DMB}$ at $\mathrm{pH} 5.5$ resulted in enhanced Dehalococcoides growth (Table 2), 10 times ( $\mathrm{pH} 5.5$ $+\mathrm{DMB}$ ) vs four times ( $\mathrm{pH} 5.5$ without DMB addition), and a higher final $\mathrm{pH}, 4.8$ ( $\mathrm{pH} 5.5+\mathrm{DMB})$ vs 4.0 ( $\mathrm{pH} 5.5$ without $\mathrm{DMB}$ addition). Interestingly, in the cultures incubated at $\mathrm{pH}$ 5.5 with $10 \mu \mathrm{M} \mathrm{DMB}$, the $\mathrm{pH}$ decreased to 4.8 as opposed to 4.0 for the cultures incubated at $\mathrm{pH} 5.5$ without DMB. This buffering effect is likely related to the documented inhibition of Sporomusa by $\mathrm{DMB},{ }^{46}$ which resulted in reduced acetogenesis. In terms of yield, at $\mathrm{pH} 6.3$ and 7.0, the yield of Dehalococcoides $\left(1.1 \pm 0.1 \times 10^{7}\right.$ cells per $\mu \mathrm{mol} \mathrm{Cl}{ }^{-}$released $)$is in the same order of magnitude as previously published yields for Dehalococcoides pure cultures, which range between $1.5 \times 10^{7}$ and $2.9 \times 10^{8}$ cells per $\mu \mathrm{mol} \mathrm{Cl^{- }}$ released, ${ }^{49}$ yet these published yields were measured in cultures generally grown with cyanocobalamin supplementation at $50 \mu \mathrm{g} / \mathrm{L}$. ${ }^{1,41}$ The 


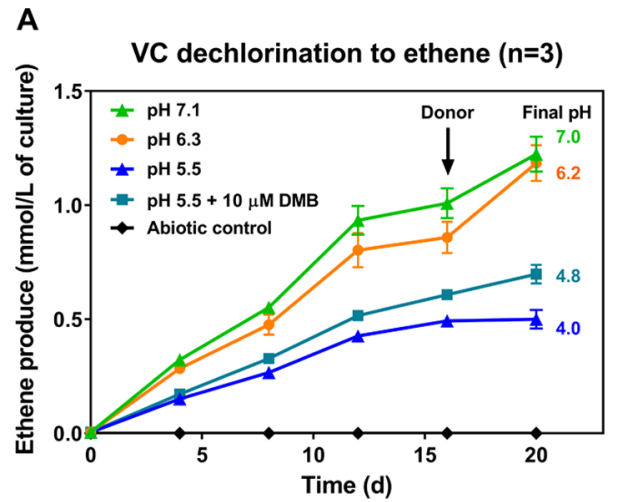

\section{B}

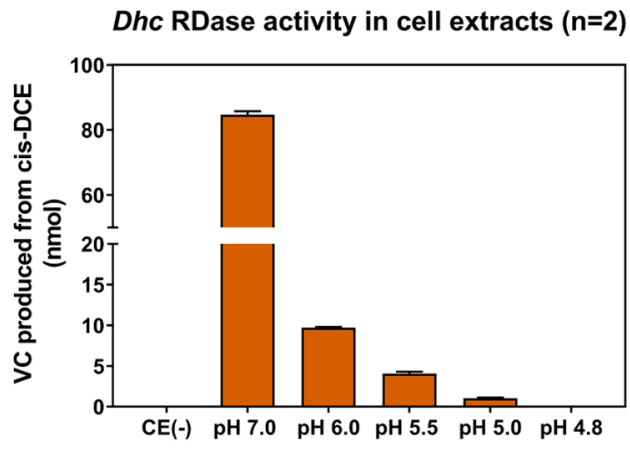

Figure 5. Effect of $\mathrm{pH}$ on the reductive dechlorination of $\mathrm{VC}$ by Dehalococcoides in $\mathrm{KB}-1$ enrichment cultures originally grown at circumneutral $\mathrm{pH}$. Dechlorination of VC to ethene in 10\% dilution transfers of the TCE/M_B12(-) enrichment culture amended with VC and methanol (A) and Dehalococcoides RDase activity in cell extracts, amended with cis-DCE, of the TCE/M_Vit(-) enrichment culture, measured at varying $\mathrm{pH}$ values (B). In (A), the initial $\mathrm{pH}$ of the transfers is shown in the figure legend and the final measured $\mathrm{pH}$ is shown next to the last measured time point; error bars represent the standard deviation of triplicate experiments. In (B), $\mathrm{CE}(-)$ stands for the control without cell extract; error bars represent the range of duplicate experiments.

Table 2. Changes in Dehalococcoides Cell Numbers per $\mathrm{mL}$ in $10 \%$ Dilution Tansfers of the TCE/M_B12(-) Enrichment Culture after the Dechlorination of VC to Ethene at Varying $\mathrm{pH}$ Values ${ }^{a}$

\begin{tabular}{lcccccc} 
& & \multicolumn{2}{c}{ Dehalococcoides cells $/ \mathrm{mL}$} & & & \\
\cline { 2 - 4 } \multicolumn{1}{c}{ starting $\mathrm{pH}$} & final $\mathrm{pH}$ & $t=0$ day mean & $t=22$ days mean & $\mathrm{Cl}^{-}$released from VC $(\mathrm{mmol})$ & yield $\left(\right.$ cells per $\mu$ mol $\mathrm{Cl}^{-}$released) & fold change \\
5.5 & 4.0 & $7.1 \pm 0.3 \times 10^{6}$ & $2.8 \pm 2.8 \times 10^{7}$ & 0.23 & $4.5 \pm 6.1 \times 10^{6}$ & 4 \\
$5.5+10 \mu \mathrm{M} \mathrm{DMB}$ & 4.8 & $7.1 \pm 0.3 \times 10^{6}$ & $6.9 \pm 0.6 \times 10^{7}$ & 0.32 & $9.7 \pm 0.9 \times 10^{6}$ & 10 \\
6.3 & 6.2 & $5.0 \pm 0.5 \times 10^{6}$ & $1.2 \pm 0.1 \times 10^{8}$ & 0.54 & $1.1 \pm 0.1 \times 10^{7}$ & 25 \\
7.1 & 7.0 & $2.6 \pm 0.6 \times 10^{6}$ & $1.3 \pm 0.2 \times 10^{8}$ & 0.55 & $1.1 \pm 0.1 \times 10^{7}$
\end{tabular}

${ }^{a}$ Measurements at $t=0$ day correspond to the mean \pm range of duplicate qPCR reactions for single DNA samples at each pH condition. Measurements at $t=22$ days correspond to the mean and standard deviation of analyses performed on triplicate experimental bottles. Corresponding dechlorination data for each $\mathrm{pH}$ is shown in Figure 5A.

reported yields for the BAV1 and GT pure Dehalococcoides cultures grown with $1 \mu \mathrm{g} / \mathrm{L}$ cyanocobalamin were $1.4 \pm 0.8 \times$ $10^{7}$ and $5.3 \pm 1.3 \times 10^{7}$ cells per $\mu \mathrm{mol} \mathrm{Cl}^{-}$release, respectively, ${ }^{14}$ which are comparable to the yield obtained in this study.

The negative impact of low $\mathrm{pH}$ on reductive dechlorination has been well documented. ${ }^{22-26,50}$ Although members of the genus Sulfurospirillum have been shown to reductively dechlorinate PCE and TCE to cis-DCE at low $\mathrm{pH}(\mathrm{pH}$ $5.5){ }^{26}$ this is the first time that growth of Dehalococcoides populations has been demonstrated at $\mathrm{pH} 5.5$ after a rapid decrease from $\mathrm{pH} 7.0$ to 5.5 (Table 2) and also after progressive decreases and long-term cultivation at $\mathrm{pH} 5.5$ (Figure 2). Thus, Dehalococcoides is likely a key player in the complete chlorinated ethene detoxification at field sites even when $\mathrm{pH}$ is below 6.0.

Dechlorination in Cell Extracts at Different $\mathrm{pH}$ Values. To gain additional insight into the effect of $\mathrm{pH}$ on the Dehalococcoides-mediated reductive dechlorination, cell extracts, harvested from the TCE/M Vit(-) enrichment culture while actively dechlorinating, were assayed at $\mathrm{pH}$ $4.75,5.0,5.5,6.0$, and 7.0. In this culture, the ratio of $16 \mathrm{~S}$ rRNA gene copies to vinyl chloride reductase gene ( $v c r A)$ copies is about $1: 1$ with $\sim 1 \times 10^{9}$ copies per $\mathrm{mL}$ of culture (qPCR data shown in Figure S8). Titanium citrate-reduced methyl viologen and cis-DCE were used as the artificial electron donor and electron acceptor, respectively; cis-DCE was chosen as a suitable acceptor because cis-DCE can be more easily and reproducibly amended as opposed to $\mathrm{VC}$, which is a gas at room temperature. The dechlorination activity of the cell extracts declined sharply from $\mathrm{pH} 7.0$ to 6.0 (Figure 5B). At $\mathrm{pH}<5.0$, the dechlorination activity was completely lost. These results are consistent with our previous findings in the $10 \%$ dilution transfers set up at $\mathrm{pH}$ 5.5: the $\mathrm{pH}$ dropped from 5.5 to 5.0 and VC dechlorination did not occur (Figure $3 \mathrm{~B}$ ). In accordance to the cell-extract assays, VcrA enzyme activity is not expected at such low $\mathrm{pH}$. From earlier studies on the catalytic mechanism of RDases, ${ }^{51,52}$ it was possible to infer that RDases would be affected at low $\mathrm{pH}$, yet the functional $\mathrm{pH}$ range of Dehalococcoides RDases had not been previously studied.

Implications for Environmental Science and Remediation. Vitamins are key molecules for various microbially mediated processes, and only a concentration of micrograms per liter is generally required. However, microbial vitamin biosynthesis has a high fitness cost, e.g., more than 30 genes are involved in the anaerobic biosynthesis pathway of cobalamin, ${ }^{53}$ and not all microorganisms can synthesize the required vitamins. Thus, mixed microbial bioaugmentation cultures that do not require vitamin amendments are highly desirable at field sites to avoid the need for adding costly vitamins. Here, we have demonstrated that complete dechlorination of TCE to ethene occurs steadily in the KB-1 enrichment cultures grown without any exogenous vitamins (Figure 1). Vitamin autotrophy in mixed microbial communities is possible if the necessary vitamin-producing populations are present and maintained during cultivation. In KB-1, these vitamin-producing populations have been maintained owing to batch cultivation, infrequent medium changes, and the use of methanol as electron donor. At field sites, small amounts of 
methanol may be available since methanol is a naturally occurring chemical released during decomposition of different plants and animal life and produced by methane oxidizing bacteria in anaerobic environments. ${ }^{54}$ If electron donors, such as lactate, emulsified vegetable oil, or molasses, are added to a field site, cobamide-producing microorganisms are likely to be present along with other microbial populations. In fact, using metagenomics sequencing, a recent study detected the presence of Acetobacterium and Eubacterium populations, which are known to produce cobalamin, after field site biostimulation with a substrate composed of vegetable oil, cane molasses, and surfactants; at the site, TCE dechlorination to ethene was observed. ${ }^{55}$ Lactate is frequently used at field sites and during cultivation of dechlorinating cultures. Acetobacterium spp. and other cobamide-producing organisms are able to use lactate as well as methanol. ${ }^{56}$ Interestingly, dechlorinating cultures grown in the absence of cobalamin using lactate as electron donor were reported before, yet incomplete dechlorination and VC accumulation occurred. ${ }^{57,58}$ In the KB-1 cultures grown without exogenous vitamins, the presence of VcrA-containing Dehalococcoides populations along with $\mathrm{DMB} /$ cobalamin-producing Acetobacterium populations results in complete dechlorination to ethene. To our knowledge, this study is the first report of complete and sustained dechlorination to ethene in dechlorinating mixed cultures grown without any exogenous vitamins.

In a mixed microbial dechlorinating culture, and likely at field sites in which reductive dechlorination occurs, the $\mathrm{pH}$ impacts the rate and extent of dechlorination. In $\mathrm{KB}-1$, the $\mathrm{pH}$ drops progressively as a result of dechlorination and acetogenesis. Our results indicate that slow dechlorination rates at low $\mathrm{pH}(<5.5)$ are mainly due to the loss of function of Dehalococcoides RDases. The activity of Dehalococcoides RDases in cell extracts is completely lost below pH 5.0 (Figure 5B). At $\mathrm{pH}<6.0$, an enzymatic limitation, and not a cobamide limitation, was found to affect the rates and extents of VC dechlorination to ethene. However, the activity is easily rescued by raising the $\mathrm{pH}$ slightly. Clearly, a $\mathrm{pH}$ above 5.0 is essential for the successful Dehalococcoides-mediated bioremediation of vinyl chloride (VC) at contaminated field sites regardless of the availability of $\mathrm{DMB} /$ cobalamin.

Our study on $\mathrm{pH}$ and cobamide dependencies in the TCEdechlorinating mixed culture KB-1 also provides a mechanistic insight into the dynamic interactions between the populations of acetogens in a dechlorinating mixed culture. Interestingly, we found that the population of Sporomusa can grow at $\mathrm{pH}<$ 5.5, while the population of Acetobacterium can only grow at $\mathrm{pH}>6.0$. Acetobacterium is a beneficial syntrophic partner for Dehalococcoides since it can provide Dehalococcoides with $\mathrm{H}_{2}$ and acetate, as well as $\mathrm{DMB} /$ cobalamin to support complete TCE dechlorination to ethene, at a physiological $\mathrm{pH}$ range (above 6.0) that closely matches the desirable $\mathrm{pH}$ range for efficient VC dechlorination to ethene; i.e., in adapted cultures, VC dechlorination rates at $\mathrm{pH} 6.0$ and $\mathrm{pH} 7.0$ were comparable. Our results demonstrate that cobalamin inhibits the growth of Sporomusa on methanol. Thus, in KB-1, Acetobacterium likely exports cobalamin as a mechanism to inhibit Sporomusa growth and compete for methanol. Interestingly, Acetobacterium and Sporomusa, organisms with an overlapping ecological role, seem to have developed different strategies for interspecies competition and have evolved to occupy different niches.

\section{ASSOCIATED CONTENT}

\section{Supporting Information}

The Supporting Information is available free of charge on the ACS Publications website at DOI: 10.1021/acs.est.9b02339.

Quantitative PCR (qPCR) primer sequences, list of samples from methanol-fed KB-1-derived enrichment cultures, calibration information for the qPCR assays, and comparison between the proteins encoded by the bza operon; figures and detailed analytical procedures including history and experiments performed, history of the VC and methanol-fed KB-1 enrichment cultures, cumulative ethene production, MS spectra of extracted cobamides, dechlorination of TCE to ethene, DMB biosynthesis operon, cell copies per $\mathrm{mL}$ of culture, and gene copy number per $\mathrm{mL}$ of culture (PDF)

\section{AUTHOR INFORMATION}

\section{Corresponding Author}

*E-mail: elizabeth.edwards@utoronto.ca.

ORCID $\odot$

Luz A. Puentes Jácome: 0000-0003-0183-4384

Po-Hsiang Wang: 0000-0001-9900-0972

M. Ahsanul Islam: 0000-0001-9585-6263

Elizabeth A. Edwards: 0000-0002-8071-338X

\section{Present Addresses}

\#Department of Chemical Engineering, Loughborough University, Loughborough LE11 3TU, U.K. (M.A.I.).

${ }^{\perp}$ Lonza Houston Inc., Houston, Texas 77047, United States (Y.X.L.).

"Earth-Life Science Institute, Tokyo Institute of Technology, Tokyo 152-8550, Japan (P.-H.W.).

\section{Funding}

This work was supported by Genome Canada, the Ontario Genomics Institute [2009-OGI-ABC-1405], the NSERC CREATE RENEW program [180804567], the Government of Ontario through the Ontario Graduate Scholarship program (OGS to L.A.P.J.), and the Ontario Research Fund INTEGRATE project [ORF-RE05-WR-01]. Support was also provided by the Government of Ontario through a Genome Ontario SPARK Research Grant.

\section{Notes}

The authors declare no competing financial interest.

${ }^{\S}$ L.A.P.J. and P.-H.W. contributed equally to this study.

\section{ACKNOWLEDGMENTS}

We would like to thank Dr Kirill Krivushin for his earlier work regarding the dominant acetogens, Sporomusa and Acetobacterium, in the KB-1 culture. We also thank Professor Frank E. Löfller and Dr Jun Yan at the University of Tennessee, Knoxville, for maintaining and supplying the pure culture of Sporomusa sp. strain KB-1 as well as providing us with the $p$ cresolyl cobamide standard. We are grateful to Nadia Morson ( $U$ of Toronto) for performing the qPCR assays to determine the copy numbers of the vinyl chloride reductase $(v c r A)$ gene in the TCE/M Vit $(-)$ culture and to Robert Flick at the BioZone Mass Spectrometry facility (U of Toronto) for assisting with the UPLC-ESI-HRMS analyses. 


\section{REFERENCES}

(1) Maymó-Gatell, X.; Chien, Y. T.; Gossett, J. M.; Zinder, S. H. Isolation of a bacterium that reductively dechlorinates tetrachloroethene to ethene. Science 1997, 276, 1568-1571.

(2) Duhamel, M.; Wehr, S. D.; Yu, L.; Rizvi, H.; Seepersad, D.; Dworatzek, S.; Cox, E. E.; Edwards, E. A. Comparison of anaerobic dechlorinating enrichment cultures maintained on tetrachloroethene, trichloroethene, cis-dichloroethene and vinyl chloride. Water Res. 2002, 36, 4193-4202.

(3) Duhamel, M.; Mo, K.; Edwards, E. A. Characterization of a highly enriched Dehalococcoides-containing culture that grows on vinyl chloride and trichloroethene. Appl. Environ. Microbiol. 2004, 70, $5538-5545$.

(4) Yang, Y.; Higgins, S. A.; Yan, J.; Simsir, B.; Chourey, K.; Iyer, R.; Hettich, R. L.; Baldwin, B.; Ogles, D. M.; Löffler, F. E. Grape pomace compost harbors organohalide-respiring Dehalogenimonas species with novel reductive dehalogenase genes. ISME J. 2017, 11, 27672780.

(5) Gantzer, C. J.; Wackett, L. P. Reductive Dechlorination Catalyzed by Bacterial Transition-Metal Coenzymes. Environ. Sci. Technol. 1991, 25, 715-722.

(6) Maymó-Gatell, X.; Tandoi, V.; Gossett, J. M.; Zinder, S. H. Characterization of an $\mathrm{H} 2$-utilizing enrichment culture that reductively dechlorinates tetrachloroethene to vinyl chloride and ethene in the absence of methanogenesis and acetogenesis. Appl. Environ. Microbiol. 1995, 61, 3928-3933.

(7) Yan, J.; Bi, M.; Bourdon, A. K.; Farmer, A. T.; Wang, P. H.; Molenda, O.; Quaile, A. T.; Jiang, N. N.; Yang, Y.; Yin, Y. C.; Simsir, B.; Campagna, S. R.; Edwards, E. A.; Löffler, F. E. Purinyl-cobamide is a native prosthetic group of reductive dehalogenases. Nat. Chem. Biol. 2018, 14, No. 8 .

(8) Crofts, T. S.; Seth, E. C.; Hazra, A. B.; Taga, M. E. Cobamide Structure Depends on Both Lower Ligand Availability and CobT Substrate Specificity. Chem. Biol. 2013, 20, 1265-1274.

(9) Yan, J.; Simsir, B.; Farmer, A. T.; Bi, M.; Yang, Y.; Campagna, S. R.; Löffler, F. E. The corrinoid cofactor of reductive dehalogenases affects dechlorination rates and extents in organohalide-respiring Dehalococcoides mccartyi. ISME J. 2016, 10, 1092-1101.

(10) Men, Y.; Seth, E. C.; Yi, S.; Crofts, T. S.; Allen, R. H.; Taga, M. E.; Alvarez-Cohen, L. Identification of specific corrinoids reveals corrinoid modification in dechlorinating microbial communities. Environ. Microbiol. 2015, 17, 4873-4884.

(11) Yi, S.; Seth, E. C.; Men, Y. J.; Stabler, S. P.; Allen, R. H.; Alvarez-Cohen, L.; Taga, M. E. Versatility in Corrinoid Salvaging and Remodeling Pathways Supports Corrinoid-Dependent Metabolism in Dehalococcoides mccartyi. Appl. Environ. Microbiol. 2012, 78, 77457752.

(12) Men, Y. J.; Seth, E. C.; Yi, S.; Allen, R. H.; Taga, M. E.; AlvarezCohen, L. Sustainable Growth of Dehalococcoides mccartyi 195 by Corrinoid Salvaging and Remodeling in Defined Lactate-Fermenting Consortia. Appl. Environ. Microbiol. 2014, 80, 2133-2141.

(13) Men, Y.; Seth, E. C.; Yi, S.; Crofts, T. S.; Allen, R. H.; Taga, M. E.; Alvarez-Cohen, L. Identification of specific corrinoids reveals corrinoid modification in dechlorinating microbial communities. Environ Microbiol 2014, 17, 4873-4884.

(14) Yan, J.; Im, J.; Yang, Y.; Löffler, F. E. Guided cobalamin biosynthesis supports Dehalococcoides mccartyi reductive dechlorination activity. Philos. Trans. R. Soc., B 2013, 368, No. 20120320.

(15) Renz, P.; Endres, B.; Kurz, B.; Marquart, J. Biosynthesis of vitamin $\mathrm{B} 12$ in anaerobic bacteria. Transformation of 5-hydroxybenzimidazole and 5-hydroxy-6-methylbenzimidazole into 5,6dimethylbenzimidazole in Eubacterium limosum. Eur. J. Biochem. 1993, 217, 1117-1121.

(16) Stupperich, E.; Eisinger, H. J.; Krautler, B. Diversity of corrinoids in acetogenic bacteria. $p$-Cresolylcobamide from Sporomusa ovata, 5-methoxy-6-methylbenzimidazolylcobamide from Clostridium formicoaceticum and vitamin B12 from Acetobacterium woodii. Eur. J. Biochem. 1988, 172, 459-464.
(17) Molenda, O.; Tang, S.; Lomheim, L.; Edwards, E. A. Eight new genomes of organohalide-respiring Dehalococcoides mccartyi reveal evolutionary trends in reductive dehalogenases. bioRxiv 2018, No. 345173.

(18) Molenda, O.; Tang, S. Q.; Lomheim, L.; Gautam, V. K.; Lemak, S.; Yakunin, A. F.; Maxwell, K. L.; Edwards, E. A. Extrachromosomal circular elements targeted by CRISPR-Cas in Dehalococcoides mccartyi are linked to mobilization of reductive dehalogenase genes. ISME J. 2019, 13, 24-38.

(19) Duhamel, M.; Edwards, E. A. Growth and yields of dechlorinators, acetogens, and methanogens during reductive dechlorination of chlorinated ethenes and dihaloelimination of 1,2dichloroethane. Environ. Sci. Technol. 2007, 41, 2303-2310.

(20) Duhamel, M.; Edwards, E. A. Microbial composition of chlorinated ethene-degrading cultures dominated by Dehalococcoides. FEMS Microbiol. Ecol. 2006, 58, 538-549.

(21) Liang, X.; Molenda, O.; Tang, S.; Edwards, E. A. Identity and Substrate Specificity of Reductive Dehalogenases Expressed in Dehalococcoides-Containing Enrichment Cultures Maintained on Different Chlorinated Ethenes. Appl. Environ. Microbiol. 2015, 81, 4626-4633.

(22) Brovelli, A.; Barry, D. A.; Robinson, C.; Gerhard, J. I. Analysis of acidity production during enhanced reductive dechlorination using a simplified reactive transport model. Adv. Water Res. 2012, 43, 1427.

(23) Duhamel, M.; Wehr, S. D.; Yu, L.; Rizvi, H.; Seepersad, D.; Dworatzek, S.; Cox, E. E.; Edwards, E. A. Comparison of anaerobic dechlorinating enrichment cultures maintained on tetrachloroethene, trichloroethene, cis-dichloroethene and vinyl chloride. Water Res. 2002, 36, 4193-202.

(24) Lacroix, E.; Brovelli, A.; Holliger, C.; Barry, D. A. Evaluation of silicate minerals for $\mathrm{pH}$ control during bioremediation: Application to chlorinated solvents. Water, Air, Soil Pollut. 2012, 223, 2663-2684.

(25) McCarty, P. L.; Chu, M.-Y.; Kitanidis, Peter K. Electron donor and $\mathrm{pH}$ relationships for biologically enhanced dissolution of chlorinated solvent DNAPL in groundwater. Eur. J. Soil Biol. 2007, 43, 276-282.

(26) Yang, Y.; Capiro, N. L.; Marcet, T. F.; Yan, J.; Pennell, K. D.; Löffler, F. E. Organohalide Respiration with Chlorinated Ethenes under Low pH Conditions. Environ. Sci. Technol. 2017, 51, 85798588.

(27) Environmental, T.Products and Services for in Situ Remediation: Vitamin B12. https://www.tersusenv.com/ bioremediation-supplements/b12 (accessed October 30, 2018).

(28) Puentes Jácome, L. A.; Edwards, E. A. A switch of chlorinated substrate causes emergence of a previously undetected native Dehalobacter population in an established Dehalococcoides-dominated chloroethene-dechlorinating enrichment culture. FEMS Microbiol. Ecol. 2017, 93, No. fix141.

(29) Wang, P. H.; Tang, S. Q.; Nemr, K.; Flick, R.; Yan, J.; Mahadevan, R.; Yakunin, A. F.; Löffler, F. E.; Edwards, E. A. Refined experimental annotation reveals conserved corrinoid autotrophy in chloroform-respiring Dehalobacter isolates. ISME J. 2017, 11, 626640.

(30) Edwards, E. A.; Grbić-Galić, D. Anaerobic Degradation of Toluene and O-Xylene by a Methanogenic Consortium. Appl. Environ. Microbiol. 1994, 60, 313-322.

(31) Islam, M. A. A Systems-Level Investigation of the Metabolism of Dehalococcoides mccartyi and the Associated Microbial Community. Ph.D. Thesis, University of Toronto: Canada, 2014.

(32) Li, Y. X. Adaptation of a Dechlorinating Culture, KB-1, to Acidic Environments. Doctoral Dissertation, University of Toronto: Canada, 2012.

(33) Wang, P. H.; Tang, S.; Nemr, K.; Flick, R.; Yan, J.; Mahadevan, R.; Yakunin, A.; Löffler, F. E.; Edwards, E. A. Refined Experimental Annotation Reveals Conserved Corrinoid Autotrophy in ChloroformRespiring Dehalobacter Isolates. ISME J 2016, 11, No. 626. 
(34) Bache, R.; Pfennig, N. Selective Isolation of Acetobacterium woodii on Methoxylated Aromatic-Acids and Determination of Growth Yields. Arch. Microbiol. 1981, 130, 255-261.

(35) Braun, M.; Gottschalk, G. Acetobacterium-Wieringae Sp-Nov, a New Species Producing Acetic-Acid from Molecular-Hydrogen and Carbon-Dioxide. Zentralbl. Bakteriol., Mikrobiol. Hyg., Abt. 1, Orig. C 1982, 3, 368-376.

(36) Eichler, B.; Schink, B. Oxidation of Primary Aliphatic-Alcohols by Acetobacterium-Carbinolicum Sp-Nov, a Homoacetogenic Anaerobe. Arch. Microbiol. 1984, 140, 147-152.

(37) Traunecker, J.; Preuß, A.; Diekert, G. Isolation and characterization of a methyl chloride utilizing, strictly anaerobic bacterium. Arch. Microbiol. 1991, 156, 416-421.

(38) Möller, B.; Ossmer, R.; Howard, B. H.; Gottschalk, G.; Hippe, H. Sporomusa, a New Genus of Gram-Negative Anaerobic-Bacteria Including Sporomusa-Sphaeroides Spec-Nov and Sporomusa ovata Spec-Nov. Arch. Microbiol. 1984, 139, 388-396.

(39) Kuhner, C. H.; Frank, C.; Griesshammer, A.; Schmittroth, M.; Acker, G.; Gossner, A.; Drake, H. L. Sporomusa silvacetica sp nov, an acetogenic bacterium isolated from aggregated forest soil. Int. J. Syst. Bacteriol. 1997, 47, 352-358.

(40) Ollivier, B.; Cordruwisch, R.; Lombardo, A.; Garcia, J. L. Isolation and Characterization of Sporomusa-Acidovorans $\mathrm{Sp}-\mathrm{Nov}$, a Methylotrophic Homoacetogenic Bacterium. Arch. Microbiol. 1985, 142, 307-310.

(41) Löffler, F. E.; Yan, J.; Ritalahti, K. M.; Adrian, L.; Edwards, E. A.; Konstantinidis, K. T.; Muller, J. A.; Fullerton, H.; Zinder, S. H.; Spormann, A. M. Dehalococcoides mccartyi gen. nov., sp nov., obligately organohalide-respiring anaerobic bacteria relevant to halogen cycling and bioremediation, belong to a novel bacterial class, Dehalococcoidia classis nov., order Dehalococcoidales ord. nov and family Dehalococcoidaceae fam. nov., within the phylum Chloroflexi. Int. J. Syst. Evol. Microbiol. 2013, 63, 625-635.

(42) Stupperich, E.; Krautler, B. Pseudo Vitamin-B12 or 5Hydroxybenzimidazolyl-Cobamide Are the Corrinoids Found in Methanogenic Bacteria. Arch. Microbiol. 1988, 149, 268-271.

(43) Guimaraes, D. H.; Weber, A.; Klaiber, I.; Vogler, B.; Renz, P. Guanylcobamide and Hypoxanthylcobamide - Corrinoids Formed by Desulfovibrio vulgaris. Arch. Microbiol. 1994, 162, 272-276.

(44) Lamm, L.; Horig, J. A.; Renz, P.; Heckmann, G. Biosynthesis of Vitamin-B12 - Experiments with the Anaerobe Eubacterium limosum and Some Labeled Substrates. Eur. J. Biochem. 1980, 109, 115-118.

(45) Hazra, A. B.; Han, A. W.; Mehta, A. P.; Mok, K. C.; Osadchiy, V.; Begley, T. P.; Taga, M. E. Anaerobic biosynthesis of the lower ligand of vitamin B-12. Proc. Natl. Acad. Sci. U.S.A. 2015, 112, 1079210797.

(46) Mok, K. C.; Taga, M. E. Growth inhibition of Sporomusa ovata by incorporation of benzimidazole bases into cobamides. J. Bacteriol. 2013, 195, 1902-1911.

(47) Stupperich, E.; Konle, R. Corrinoid-dependent methyl transfer reactions are involved in methanol and 3, 4-dimethoxybenzoate metabolism by Sporomusa ovata. Appl. Environ. Microbiol. 1993, 59, $3110-3116$.

(48) Stupperich, E.; Aulkemeyer, P.; Eckerskorn, C. Purification and characterization of a methanol-induced cobamide-containing protein from Sporomusa ovata. Arch. Microbiol. 1992, 158, 370-373.

(49) Löffler, F. E.; Yan, J.; Ritalahti, K. M.; Adrian, L.; Edwards, E. A.; Konstantinidis, K. T.; Muller, J. A.; Fullerton, H.; Zinder, S. H.; Spormann, A. M. Dehalococcoides mccartyi gen. nov., sp. nov., obligate organohalide-respiring anaerobic bacteria, relevant to halogen cycling and bioremediation, belong to a novel bacterial class, Dehalococcoidetes classis nov., within the phylum Chloroflexi. Int. J. Syst. Evol. Microbiol. 2012, 63, 625-635.

(50) Yang, Y.; Capiro, N. L.; Yan, J.; Marcet, T. F.; Pennell, K. D.; Löffler, F. E. Resilience and recovery of Dehalococcoides mccartyi following low $\mathrm{pH}$ exposure. FEMS Microbiol. Ecol. 2017, 93, No. fix130.

(51) Payne, K. A. P.; Quezada, C. P.; Fisher, K.; Dunstan, M. S.; Collins, F. A.; Sjuts, H.; Levy, C.; Hay, S.; Rigby, S. E. J.; Leys, D.
Reductive dehalogenase structure suggests a mechanism for B12dependent dehalogenation. Nature 2015, 517, No. 513.

(52) Bommer, M.; Kunze, C.; Fesseler, J.; Schubert, T.; Diekert, G.; Dobbek, H. Structural basis for organohalide respiration. Science 2014, $346,455-458$

(53) Roth, J. R.; Lawrence, J. G.; Rubenfield, M.; Kiefferhiggins, S.; Church, G. M. Characterization of the Cobalamin (Vitamin-B12) Biosynthetic Genes of Salmonella-Typhimurium. J. Bacteriol. 1993, $175,3303-3316$.

(54) Lieberman, R. L.; Rosenzweig, A. C. Biological methane oxidation: Regulation, biochemistry, and active site structure of particulate methane monooxygenase. Crit. Rev. Biochem. Mol. Biol. 2004, 39, 147-164.

(55) Kao, C. M.; Liao, H. Y.; Chien, C. C.; Tseng, Y. K.; Tang, P.; Lin, C. E.; Chen, S. C. The change of microbial community from chlorinated solvent-contaminated groundwater after biostimulation using the metagenome analysis. J. Hazard. Mater. 2016, 302, 144150 .

(56) Schoelmerich, M. C.; Katsyv, A.; Sung, W.; Mijic, V.; Wiechmann, A.; Kottenhahn, P.; Baker, J.; Minton, N. P.; Muller, V. Regulation of lactate metabolism in the acetogenic bacterium Acetobacterium woodii. Environ. Microbiol. 2018, 20, 4587-4595.

(57) Men, Y. J.; Yu, K.; Baelum, J.; Gao, Y.; Tremblay, J.; Prestat, E.; Stenuit, B.; Tringe, S. G.; Jansson, J.; Zhang, T.; Alvarez-Cohen, L. Metagenomic and Metatranscriptomic Analyses Reveal the Structure and Dynamics of a Dechlorinating Community Containing Dehalococcoides mccartyi and Corrinoid-Providing Microorganisms under Cobalamin-Limited Conditions. Appl. Environ. Microbiol. 2017, 83, No. e03508-16.

(58) Men, Y. J.; Lee, P. K. H.; Harding, K. C.; Alvarez-Cohen, L. Characterization of four TCE-dechlorinating microbial enrichments grown with different cobalamin stress and methanogenic conditions. Appl. Microbiol. Biotechnol. 2013, 97, 6439-6450. 